\title{
Fibroblast Growth Factor Receptor-2 Polymorphism rs2981582 is Correlated With Progression-free Survival and Overall Survival in Patients With Metastatic Clear-cell Renal Cell Carcinoma Treated With Sunitinib
}

Maxime Vanmechelen, ${ }^{1}$ Diether Lambrechts, ${ }^{2,3}$ Thomas Van Brussel, ${ }^{2,3}$ Annelies Verbiest, ${ }^{1}$ Gabrielle Couchy,${ }^{4}$ Patrick Schöffski, ${ }^{1}$ Herlinde Dumez, ${ }^{1}$ Philip R. Debruyne, ${ }^{5,6}$ Evelyne Lerut, ${ }^{7}$ Jean-Pascal Machiels, ${ }^{8}$ Vincent Richard, ${ }^{9}$ Maarten Albersen, ${ }^{10}$ Vincent Verschaeve, ${ }^{11}$ Stéphane Oudard, ${ }^{12}$ Arnaud Méjean, ${ }^{13}$ Pascal Wolter, ${ }^{14}$ Jessica Zucman-Rossi, ${ }^{4}$ Benoit Beuselinck ${ }^{1,4}$

\section{Abstract}

We describe a potential biomarker associated with progression-free survival and overall survival on sunitinib in metastatic clear-cell renal cell carcinoma. rs2981582 is a polymorphism in the fibroblast growth factor receptor 2. In our series of 154 patients treated with sunitinib, the TT-variant, present in $13 \%$ of the patients, was associated with shorter progression-free survival and overall survival.

Background: There are no validated markers that predict response or resistance in patients with metastatic clear-cell renal cell carcinoma (mccRCC) treated with vascular endothelial growth factor receptor tyrosine kinase inhibitors such as sunitinib and pazopanib. Recently, single nucleotide polymorphism (SNP) rs2981582 in Fibroblast Growth Factor Receptor 2 (FGFR2) was found to be associated with clinical outcome in patients with mccRCC treated with pazopanib and sunitinib. We aimed to validate these findings in patients treated with sunitinib. Materials and Methods: Germline DNA was collected in patients with mccRCC starting first-line systemic therapy with sunitinib. SNP rs2981582 in FGFR2 C>T was genotyped. Association of the genotype with response rate, tumor shrinkage, median progression-free survival (mPFS), and median overall survival (mOS) was studied. Results: We collected clinical data from 154 patients with available germline DNA. Baseline prognostic markers were well-balanced between both subgroups. Patients with the TT genotype had a poorer outcome compared with patients with the CT/CC genotype. The median shrinkage of selected tumor target lesions during treatment with sunitinib was $-16 \%$ versus $-31 \%(P=$ .002), mPFS was 8 versus 15 months $(P=.0007)$, and mOS was 22 versus 33 months $(P=.04)$, respectively. On multivariate analysis, rs2981582 remained an independent predictor of PFS (hazard ratio, 2.858; 95\% confidence interval, 1.659-4.923; $P<.0001$ ) and OS (hazard ratio, 1.795; 95\% confidence interval, $1.003-3.212 ; P=.049$ ).

\footnotetext{
${ }^{1}$ Department of General Medical Oncology, University Hospitals Leuven, Leuven Cancer Institute

${ }^{2}$ Laboratory for Translational Genetics, Department of oncology, KU Leuven, Leuven, Belgium

${ }^{3}$ Vesalius Research Center, VIB, Leuven, Belgium

${ }^{4}$ Inserm UMR1162 Génomique Fonctionnelle des Tumeurs Solides, Université Paris-5 René Descartes, Paris, France

${ }^{5}$ Department of Medical Oncology, AZ Groeninge, Kortrijk, Belgium

${ }^{6}$ Faculty of Health, Social Care, and Education, Anglia Ruskin University, Chelmsford, UK

${ }^{7}$ Department of Pathology, University Hospitals Leuven, KU Leuven, Leuven, Belgium

${ }^{8}$ Department of Medical Oncology, Cliniques Universitaires Saint-Luc, UCLouvain,

Bruxelles, Belgium

${ }^{9}$ Department of Medical Oncology, CHU Ambroise Paré, Mons, Belgium
}

\begin{tabular}{l}
\hline${ }^{10}$ Department of Urology, University Hospitals Leuven, KU Leuven, Leuven, Belgium \\
${ }^{11}$ Department of Medical Oncology, Grand Hôpital de Charleroi, Charleroi, Belgium \\
${ }^{12}$ Department of Medical Oncology, Georges Pompidou European Hospital \\
${ }^{13}$ Department of Urology, Georges Pompidou European Hospital, Université Paris-5 \\
René Descartes, Paris, France \\
${ }^{14}$ Department of Medical Oncology, St. Nikolaus-Hospital Eupen, Eupen, Belgium \\
\hline
\end{tabular}

Submitted: Jul 2, 2018; Revised: Nov 8, 2018; Accepted: Nov 8, 2018

Address for correspondence: Dr Benoit Beuselinck, MD, PhD, Leuven Cancer Institute, KU Leuven, Herestraat 49, 3000 Leuven, Belgium

E-mail contact: benoit.beuselinck@uzleuven.be 


\section{FGFR2 Polymorphism as Biomarker for Outcome on Sunitinib}

Conclusion: Polymorphism rs2981582 in FGFR2 is correlated to PFS and OS in patients with mccRCC treated with sunitinib. Prospective validation of the impact of this SNP is warranted.

Clinical Genitourinary Cancer, Vol. ш, No. ш, 1-10 (c) 2018 Elsevier Inc. All rights reserved.

Keywords: Angiogenesis inhibitor, Biomarker, Outcome, Prognosis, Single nucleotide polymorphisms

\section{Introduction}

Clear-cell renal cell carcinoma (ccRCC) is characterized by ubiquitous loss of a functional Von Hippel Lindau protein, caused by mutation, promotor hypermethylation, or loss of heterozygosity. ${ }^{1,2}$ This results in an increase in hypoxia inducible factor, ${ }^{3}$ and, among other effects, subsequent activation of vascular endothelial growth factor (VEGF)-dependent angiogenesis. Targeted therapies directed against the VEGF pathway are the current standard of care as first-line treatment of patients with metastatic (m) ccRCC. ${ }^{4,5}$ Apart from bevacizumab, which is an anti-VEGF antibody, these therapies are tyrosine kinase inhibitors (TKIs) such as sunitinib, pazopanib, axitinib, cabozantinib, or sorafenib, inhibiting VEGFreceptors (VEGFRs), and other molecular targets. Sunitinib and pazopanib are most often used in first-line therapy. ${ }^{6,7}$ Clinical responses are highly variable, and even patients who initially respond well will ultimately develop secondary resistance. ${ }^{8}$ Unfortunately, there are no validated predictive biomarkers for response or resistance in patients with mccRCC treated with VEGFR-TKIs.

The VEGF-dependent pro-angiogenetic pathway targeted by these therapies has been the object of several studies searching for predictive biomarkers. Von Hippel Lindau mutations are not correlated with efficacy. ${ }^{9}$ On a transcriptomic level, upregulation of angiogenesis-related genes has been associated with better response to VEGFR-TKIs. ${ }^{10-13}$ Finally, several studies have linked single nucleotide polymorphisms (SNPs) in genes encoding proteins in the VEGF pathway with outcome in patients with mccRCC treated with sunitinib. ${ }^{14-17}$ However, validation of these findings in independent patient series has been challenging. ${ }^{18,19}$

Activation of VEGF-independent neo-angiogenesis, for instance through the FGFR pathway, is suggested as one of the putative mechanisms of resistance to VEGF-directed therapy. ${ }^{20}$ When the VEGF-dependent pro-angiogenetic pathway is blocked by VEGFRTKIs, neo-angiogenesis and tumor growth could continue through the FGFR pathway. Therefore, FGFR blockers such as dovitinib and lenvatinib have been tested in mccRCC. ${ }^{21,22}$ The TT variant of $\mathrm{SNP}$ rs2981582C $>\mathrm{T}$ in FGFR2 has been associated with increased FGFR2 gene expression in breast cancer cell lines. ${ }^{23}$

The possible impact of SNP rs2981582 in FGFR2 on outcome in patients with mRCC treated with VEGFR-TKIs was previously shown in patients treated with pazopanib. In 380 patients treated in first-line with pazopanib in 3 studies, among them the pazopanib pivotal trial, ${ }^{7} \mathrm{Xu}$ et al showed the negative impact of the TT variant in rs2981582 on progression-free survival (PFS) $(P=.053) .^{24}$ In 241 patients included in the pazopanib pivotal trial, rs2981582 was associated with median overall survival (mOS) (hazard ratio [HR], 1.40; 95\% confidence interval [CI], 1.09-1.81; $P=.008),{ }^{25}$ favoring CT/CC carriers. We previously published the impact of rs 2981582 on outcome in patients with mRCC treated with sunitinib. We compared outcome in 23 patients with the CC genotype and 12 with the TT genotype. The median PFS (mPFS) was 14 versus 7.5 months, respectively $(P=.012)$, but no impact on OS was shown. ${ }^{17}$ The outcome of the CT carriers was not studied, because our analysis at that moment was based on an abstract of Xu et al comparing OS in CC versus TT carriers treated with pazopanib.

The aim of the present study was to validate the impact of SNP rs 2981582 in a larger series of patients with mccRCC treated with sunitinib as first-line VEGF-targeted therapy and to study more in detail the impact of the 3 different genotype combinations (CC, CT, and TT) on outcome.

\section{Materials and Methods}

For this retrospective study, germ-line DNA samples were collected in the "CIT-rein" kidney tumor bank (frozen normal kidney tissue), in patients treated at the University Hospitals Leuven (peripheral blood samples) and in patients included in the Belgian multicentric METASUN (METAbolimics in SUNitinib treated renal cell carcinoma patients) study (peripheral blood samples). The French-Belgian multicentric CIT-rein kidney tumor bank contains frozen kidney tumor samples collected at 20 academic hospitals in Belgium and France. Eligible patients could have received cytokines as systemic treatment for kidney tumors before starting sunitinib as a monotherapy. Patients who received previous treatment with any other targeted therapy before starting sunitinib were excluded. The study was approved by the medical ethics review boards of all participating institutions, and signed informed consent was obtained from all patients. DNA was isolated at INSERM U1162 in Paris, France, from fresh frozen normal kidney tissue sampled in the nephrectomy specimen using the Qiaquick extraction kit (Qiagen, Valencia, CA) and quantified by fluorometry (Fluoroskan Thermo Labsystems, Cergy-Pontoise, France). DNA was isolated from peripheral blood at the Vesalius Research Center in Leuven, Belgium, with the Qiagen DNA kit (Qiagen), and final DNA concentration was quantified with Nanodrop (Nanodrop, Wilmington, DE). High-throughput SNP genotyping was performed at the Vesalius Research Center in Leuven, Belgium, using the Sequenom MassArray platform (Sequenom, San Diego, CA). ${ }^{26}$ Genotyping analysis was performed by investigators blinded for the clinical data.

All patients were treated in routine clinical practice. The treating oncologist could change treatment approach concerning drug schedule, dose-reduction policy, and timing of radiologic assessments in accordance with current local practice guidelines. Computed tomography of the thorax-abdomen was performed every 2 cycles of sunitinib, in most cases. All patients started their sunitinib therapy at the standard sunitinib dose of $50 \mathrm{mg} /$ day for 4 weeks on, 2 weeks off. Commonly used prognostic factors were 
assessed: sarcomatoid dedifferentiation, presence of bone metastases, and the variables included in the International Metastatic Renal Cell Carcinoma Database Consortium (IMDC) score: baseline neutrophil count, baseline platelets and hemoglobin, calcium, time between initial diagnosis and start of systemic therapy, and Karnofsky performance status. ${ }^{27}$ Primary kidney tumors were also classified according to the molecular ccRCC 1 to 4 classification as described previously. ${ }^{13}$ This expression profile-based classification has a prognostic value in patients treated with metastasectomy ${ }^{28}$ and a predictive value in patients treated with sunitinib ${ }^{13}$ or pazopanib. $^{29}$

Clinical data were collected at 19 different sites in France and Belgium. The main objective of the study was to investigate the impact of rs 2981582 on outcome in patients with mccRCC treated with sunitinib and to investigate whether this impact would be prognostic or predictive.

The primary endpoints of the study were PFS, response rate (RR), and tumor shrinkage. The secondary endpoint was OS. In fact, OS can be influenced by sequential therapies administered after first-line sunitinib, particularly immune checkpoint inhibitors, that have an activity mechanism thought to be independent of angiogenesis. We defined PFS as the interval between the first day on treatment with sunitinib and the date of radiologic progressive disease or death. Patients who had not progressed at database closure were censored at last follow-up. OS was defined as the interval between the first day on sunitinib and the date of death or last date of follow-up. Objective response was assessed by treating doctors using Response Evaluation Criteria in Solid Tumors (RECIST). We studied not only the impact of RECIST categories (complete response $[\mathrm{CR}]$, partial response $[\mathrm{PR}]$, stable disease [SD], or progressive disease $[\mathrm{PD}]$ ), but also the precise percentage of RECIST tumor shrinkage compared with baseline, whenever available. The precise percentage of tumor shrinkage can give additional and more precise information compared with the RECIST categories CR, PR, $\mathrm{SD}$, and PD. On one hand, although the difference between a SD with $29 \%$ of tumor shrinkage and a PR with $31 \%$ of shrinkage is not an important difference in shrinkage, patients are classified in another response category. On the other hand, 2 patients with tumor shrinkage of $35 \%$ and $95 \%$ will both be classified in the PR group, but the response has been more important in the latter case.

The impact of rs2981582 was studied in a discovery and a validation cohort. The discovery cohort was composed of the 88 patients included in our previous publication, ${ }^{17}$ in which we reported the impact of SNPs in several genes such as VEGFR3, ABCB1, NR1/3, NR1/2, PDGFRA, and FGFR2. However, in this previous publication, concerning FGFR2, we only reported outcome in CC carriers $(\mathrm{n}=23)$ compared with TT carriers $(\mathrm{n}=$ 12), because we aimed to replicate data presented in 2011 in an abstract by $\mathrm{Xu}$ et al comparing OS in CC versus TT carriers treated with pazopanib. The outcome for CT carriers was not reported in this previous study. Now, we aimed to study more in detail the impact of the 3 different genotype combinations (CC, CT, and TT) on outcome in our series of patients genotyped in 2011. The validation cohort was composed of new patient samples genotyped from 2013 forward.

All patient characteristics were tested in univariate fashion to study the association with mPFS and mOS using Kaplan-Meier estimates and in a multivariate model using Cox proportional hazards. The Fisher exact test was used to compare percentages, and the Student $t$ test was applied for comparison of tumor shrinkage between carriers of different genotypes. All variables that did correlate with PFS and OS on univariate analysis with a $P$-value of $<.2$ were included in the multivariate analysis. Results with a $P$-value of $<.05$ were considered as significant in the univariate and multivariate analyses. Statistical analyses were conducted using GraphPad Prism 5 (GraphPad Software, La Jolla, CA) and XLSTAT software (Addinsoft, Paris, France).

\section{Results}

\section{Included Patients}

We included 154 patients who started sunitinib between November 2005 and July 2016 and closed the follow-up database in September 2017. This series included the 35 patients assessed in the project published earlier. ${ }^{17}$ Table 1 shows the clinical characteristics of patients included in this project. The mean age at diagnosis was 59 years (range, 30-80 years), with a male predominance (71\%). The majority of patients were of Caucasian origin. Fifty-five percent had Fuhrman grade IV ccRCCs on the initial nephrectomy specimen or biopsy. According to IMDC prognostic criteria, 15\% of patients were categorized into the favorable risk group, $61 \%$ had intermediate risk, and $24 \%$ had poor risk. In 85 patients, the primary tumor was classified according to the ccRCC 1 to 4 classification. At the time of final analysis, 121 (79\%) patients had reached progression, and $108(70 \%)$ had died. The median follow-up was 47.5 months (range, 2.0-239.0 months) after the start of sunitinib. The global mPFS was 13 months and mOS 30 months. Best RECIST response assessment was available in 147 patients. Eleven (7\%) of 147 patients had a CR, 60 (41\%) of 147 patients a PR, 53 (36\%) of 147 patients an SD, and $23(16 \%)$ of 147 patients a PD as best response. In 6 patients, there was a clinical benefit, but response assessment was poorly defined in the medical records, and as a consequence, it was unclear whether the best response was either PR or SD in these 6 patients. One patient died after 1 month of treatment with sunitinib. These results are comparable to phase III and expanded access response data. ${ }^{4,30}$ The precise percentage of RECIST tumor shrinkage was available in 103 patients. Forty-four (29\%) of 154 patients carried the FGFR2 rs2981582 CC genotype, $90(58 \%)$ of 154 patients were heterozygous (CT genotype), and the remaining 20 patients (13\%) had $2 \mathrm{~T}$ alleles. The allele distribution was as follows: $\mathrm{T}$ was present in $42.2 \%$ and $\mathrm{C}$ in $57.8 \%$. This is coherent with the minor allele frequency reported on dbSNP (https://www.ncbi.nlm.nih.gov/SNP/) (45.6\%). Around 75\% of the patients received a second-line therapy. Among them, 29 patients were treated with immune checkpoint inhibitors ( 2 in the TT group and 27 in the CC + CT group).

\section{Discovery Cohort}

The discovery cohort was composed of the patient with samples genotyped in 2011: 12 TT, 52 CT, and 23 CC carriers. The genotype was unknown in 1 patient. The mPFS was 8,19 , and 16 months, respectively, in TT, CT, and CC carriers $(P=.03)$. As Kaplan-Meier curves for PFS were overlapping in CT and CC carriers, we pooled CT and CC carriers. The mPFS was 8 versus 18 months in the TT and CC/CT carriers, respectively (HR, 0.3062; 


\section{FGFR2 Polymorphism as Biomarker for Outcome on Sunitinib}

Table 1 Patient Characteristics at Diagnosis and at the Start of Sunitinib Treatment and Baseline Clinical and Biochemical Parameters Associated With PFS and OS

\begin{tabular}{|c|c|c|c|c|}
\hline Characteristics & $\begin{array}{c}\text { Total (N=154), } \\
\mathrm{n} / \mathrm{N}(\%)\end{array}$ & $\mathrm{CC}+\underset{\mathrm{n} / \mathrm{N}(\%)}{\mathrm{CT}}(\mathrm{N}=134)$ & $\begin{array}{l}\mathrm{TT}(\mathrm{N}=20) \\
\mathrm{n} / \mathrm{N}(\%)\end{array}$ & $P$ Value \\
\hline \multicolumn{5}{|l|}{ At initial diagnosis } \\
\hline Male & 109/154 (71) & 98/134 (73) & $11 / 20(55)$ & .12 \\
\hline Mean age, y (range) & $59(30-80)$ & $59(35-78)$ & $62(30-80)$ & \\
\hline \multicolumn{5}{|l|}{ Ethnic origin } \\
\hline Caucasian & 139/154 (90) & 122/134 (91) & $17 / 20(85)$ & .42 \\
\hline Unknown & 15/154 (10) & 12/134 (9) & 3/20 (15) & .42 \\
\hline M1 (synchronous metastases) & 85/150 (57) & 73/131 (56) & 12/19 (63) & .63 \\
\hline \multicolumn{5}{|l|}{ Fuhrman } \\
\hline Grade 4 & 82/148 (55) & $72 / 128(56)$ & $10 / 20(50)$ & .64 \\
\hline \multicolumn{5}{|l|}{ Sarcomatoid dedifferentiation } \\
\hline$\geq 25 \%$ & 5/138 (4) & 4/120 (3) & $1 / 18(6)$ & .51 \\
\hline \multicolumn{5}{|l|}{ At the start of sunitinib } \\
\hline \multicolumn{5}{|l|}{ Karnofsky performance status } \\
\hline$\leq 70$ & 25/153 (16) & 23/133 (17) & 2/20 (10) & .53 \\
\hline \multicolumn{5}{|l|}{ Neutrophils } \\
\hline$>7800 / \mathrm{mm}^{3}$ & 70/151 (46) & $58 / 131(44)$ & $12 / 20(60)$ & .23 \\
\hline \multicolumn{5}{|l|}{ Platelets } \\
\hline$>450,000 / \mathrm{mm}^{3}$ & 27/153 (18) & 24/133 (18) & 3/20 (15) & 1.00 \\
\hline \multicolumn{5}{|l|}{ Hemoglobin } \\
\hline Low (<12 g/dL [women] or < $14 \mathrm{~g} / \mathrm{dL}$ [men]) & $61 / 153(40)$ & $51 / 133(38)$ & $10 / 20(50)$ & .34 \\
\hline \multicolumn{5}{|l|}{ LDH } \\
\hline$>1.5$ ULN & 9/149 (6) & 5/130 (4) & 4/19 (21) & .02 \\
\hline \multicolumn{5}{|l|}{ Corrected calcium } \\
\hline$>10 \mathrm{mg} / \mathrm{dL}$ & $11 / 112(10)$ & 11/98 (11) & 0/14 (0) & .35 \\
\hline \multicolumn{5}{|l|}{ Time from nephrectomy to systemic treatment, mos } \\
\hline$<12$ & 100/153 (65) & $84 / 133(63)$ & $16 /(80)$ & .21 \\
\hline Immunotherapy before sunitinib & 27/153 (18) & 25/134 (19) & 2/19 (11) & .53 \\
\hline \multicolumn{5}{|l|}{ Site of metastasis } \\
\hline Lung & 116/154 (75) & 102/134 (76) & 14/20 (70) & .58 \\
\hline Liver & 31/154 (20) & 26/134 (19) & 5/20 (25) & .56 \\
\hline Bone & 55/154 (36) & 48/134 (36) & 7/20 (35) & 1.00 \\
\hline Brain & 13/154 (8) & 12/134 (9) & $1 / 20(5)$ & 1.00 \\
\hline \multicolumn{5}{|l|}{ Molecular ccRCC 1-4 classification } \\
\hline ccRCC1 & 30/85 (35) & 26/73 (36) & 4/12 (33) & .88 \\
\hline ccRCC2 & $39 / 85(46)$ & $32 / 73(44)$ & 7/12 (58) & .35 \\
\hline ccRCC3 & $3 / 85(4)$ & $3 / 73(4)$ & 0/12 (0) & .47 \\
\hline ccRCC4 & 13/85 (15) & $12 / 73(16)$ & 1/12 (8) & .47 \\
\hline \multicolumn{5}{|l|}{ IMDC prognosis } \\
\hline Favorable & 22/150 (15) & 20/132 (15) & 2/18 (11) & 1.00 \\
\hline Intermediate & $92 / 150(61)$ & 80/132 (61) & 12/18 (67) & .80 \\
\hline Poor & 36/150 (24) & 32/132 (24) & 4/18 (22) & 1.00 \\
\hline \multicolumn{5}{|l|}{ Subsequent therapy upon progression on sunitinib } \\
\hline Sunitinib ongoing & 6/98 (6) & $6 / 87(7)$ & $0 / 11(0)$ & \\
\hline \multicolumn{5}{|l|}{ Second-line therapy } \\
\hline Axitinib & $27 / 98(28)$ & 25/87 (29) & 2/11 (18) & \\
\hline Cabozantinib & 2/98 (2) & 2/87 (2) & 0/11 (0) & \\
\hline Everolimus & 22/98 (22) & 18/87 (21) & 4/11 (36) & \\
\hline Nivolumab & 9/98 (9) & 8/87 (9) & 1/11 (9) & \\
\hline
\end{tabular}


Table 1 Continued

\begin{tabular}{|c|c|c|c|c|}
\hline Characteristics & $\begin{array}{c}\text { Total }(\mathrm{N}=154), \\
\mathrm{n} / \mathrm{N}(\%)\end{array}$ & 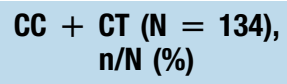 & $\begin{array}{c}\text { TT }(\mathrm{N}=20), \\
\mathrm{n} / \mathrm{N}(\%)\end{array}$ & $P$ Value \\
\hline Pazopanib & 3/98 (3) & 3/87 (3) & $0 / 11(0)$ & \\
\hline Sorafenib & $8 / 98(8)$ & $8 / 87(9)$ & $0 / 11(0)$ & \\
\hline Temsirolimus & $1 / 98(1)$ & $1 / 87(1)$ & 0/11 (0) & \\
\hline Experimental treatment & 2/98 (2) & 2/87 (2) & 0/11 (0) & \\
\hline All & 74/98 (74) & $67 / 87(77)$ & $7 / 11$ (63) & \\
\hline Palliative/died & 18/98 (18) & $14 / 87(16)$ & 4/11 (36) & \\
\hline Not available & 56 & 47 & 9 & \\
\hline
\end{tabular}

Bold value indicates significance.

Abbreviations: IMDC = The International Metastatic Renal Cell Carcinoma Database Consortium; LDH = lactate dehydrogenase activity; ULN = upper limit of normal.

95\% CI, $0.1326-0.7071 ; P=.006)$. The mOS was 23 versus 31 months in the TT and CT/CC carriers, respectively (HR, 0.6320; 95\% CI, 0.3055-1.307; $P=.22$ ) (Figure 1).

\section{Validation Cohort}

The validation cohort was composed of 67 new patients, genotyped from 2013 forward: 7 TT carriers, 38 CT carriers, and 22 CC carriers. The mPFS was 6,12 , and 12 months, respectively, in TT, $\mathrm{CT}$, and CC carriers $(P=.06)$. Again, Kaplan-Meier curves for PFS were overlapping in CC and CT carriers. When CT and CC carriers were pooled, the mPFS was 6 versus 12 months for TT and CT/CC carriers, respectively (HR, 0.2363; 95\% CI, 0.07142-0.7819; $P=.02)$. The mOS was 13 versus 34 months in the TT and CT/CC carriers (HR, 0.2444; 95\% CI, 0.06865-0.8703; $P=.03$ ) (Figure 1).

\section{Total Cobort}

In the total cohort, the mPFS was 8 and 15 months for TT and CT/CC carriers, respectively $(P=.0007)$. The mOS was 22 and 33 months for the TT and CT/CC carriers, respectively $(P=.04)$ (Figure 1). The PR rate was $37 \%$ in patients with the TT genotype compared with $50 \%$ in patients with the CT/CC genotype. This difference was not significant. CRs $(\mathrm{n}=11)$ were only noticed in the CT/CC genotype subgroup. Median tumor shrinkage was $-16 \%$ for patients with the TT genotype versus $-31 \%$ for patients with the CT/CC genotype $(P=.002)$ (Figure 2$)$.

When comparing the 3 different genotypes separately, the mPFS was 8,15 , and 14 months for patients with the TT, CT, and CC genotype, respectively $(P=.005)$. The curves of CT and CC carriers were overlapping (Figure 3). The PR rate was 35\%, 51\%,

\section{Figure 1 Kaplan-Meier Estimates Showing the Impact of rs2981582 on Progression-free Survival and Overall Survival in the Discovery Cohort, the Validation Cohort, and the Total Patient Series}

\section{PFS (\%) DISCOVERY COHORT}

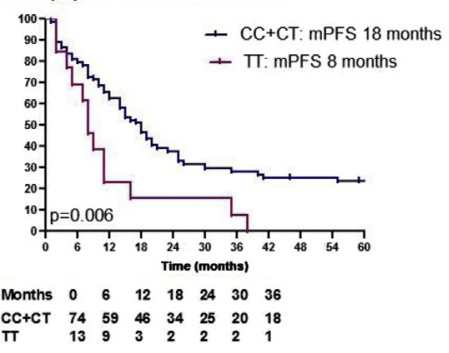

OS (\%) DISCOVERY COHORT

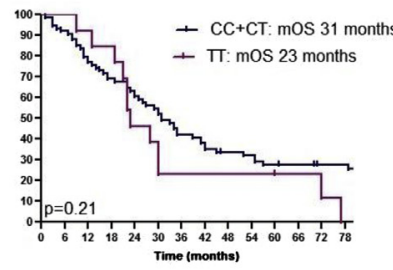

Months $\begin{array}{lllllllll}0 & 6 & 12 & 18 & 24 & 30 & 36 & 42\end{array}$ $\begin{array}{lllllllll}\mathrm{CC}+\mathrm{CT} & 74 & 68 & 59 & 49 & 45 & 39 & 30 & 27 \\ \pi \mathrm{T} & 13 & 13 & 12 & 11 & 6 & 5 & 3 & 3\end{array}$

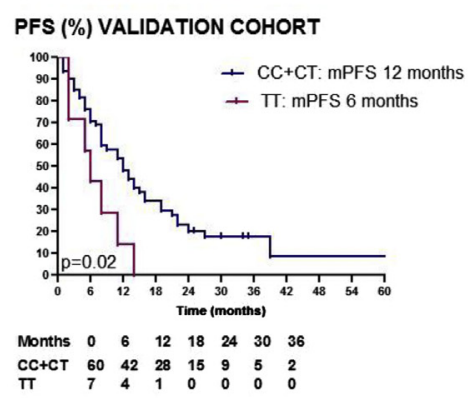

OS (\%) VALIDATION COHORT

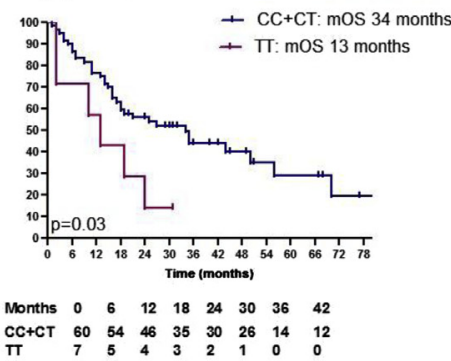

\section{PFS (\%) TOTAL SERIES}

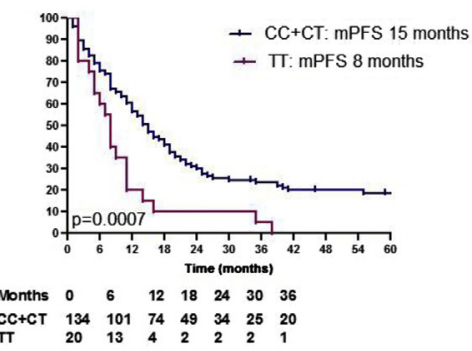

OS (\%) TOTAL SERIES

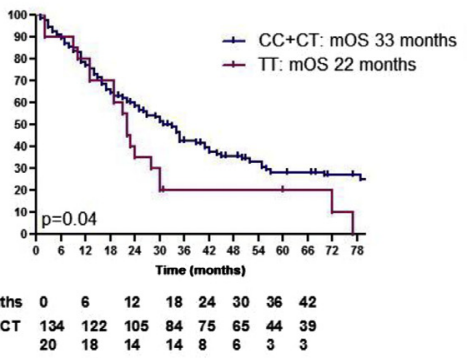

Abbreviations: $O S=$ overall survival; PFS = progression-free survival. 


\section{FGFR2 Polymorphism as Biomarker for Outcome on Sunitinib}

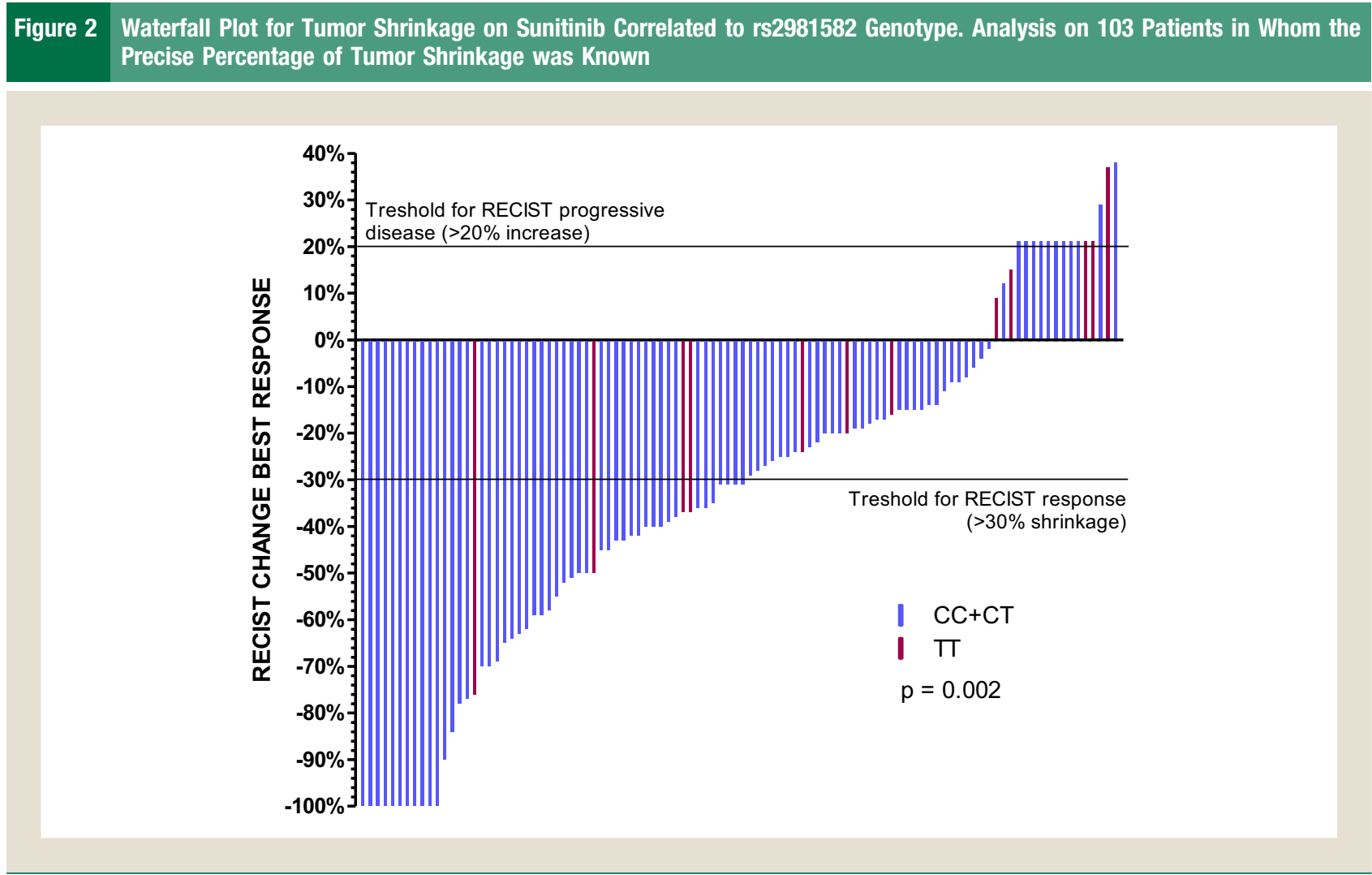

Abbreviation: RECIST $=$ Response Evaluation Criteria In Solid Tumours.

and 48\%, for TT, CT, and CC carriers, respectively; however, these differences were not significantly different. PD as best response was observed in $21 \%, 13 \%$, and $16 \%$ of the patients, respectively. The median percentage of tumor shrinkage was $-16 \%,-35 \%$, and $-28 \%$ for patients with the TT, CT, and CC genotype, respectively $(P=.09)$. The mOS was 22,35 , and 19 months, respectively $(P=.04)$. The OS curve of the CC carriers lied in between the curves of the CT carriers and TT carriers (Figure 3).

\section{Multivariate Analysis}

In the multivariate analysis, we included the commonly used prognostic markers that were significant on univariate analysis (the presence of bone metastases, baseline neutrophil count, baseline platelet count, sarcomatoid dedifferentiation, Karnofsky performance status, baseline hemoglobin levels, baseline lactate dehydrogenase [LDH] activity, and time between nephrectomy to systemic therapy $<12$ months [Table 2]). Table 1 shows that all baseline

\section{Figure 3 Kaplan-Meier Estimates Showing the Impact of the 3 Genotypes (CC, CT, AND TT) of rs2981582 on Progression-free Survival and Overall Survival}
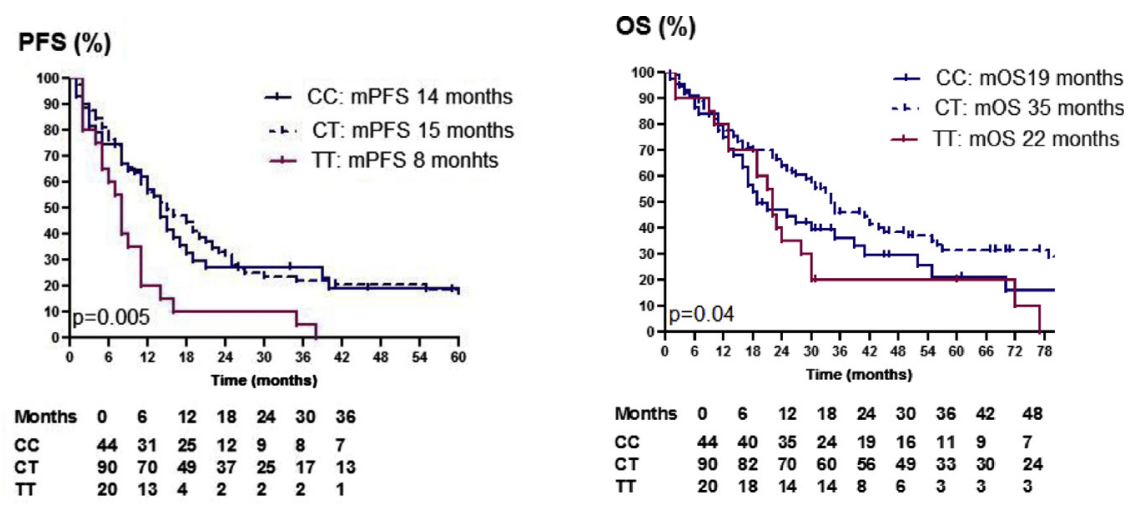

Abbreviations: $\mathrm{m}=$ median; $\mathrm{OS}=$ overall survival; $\mathrm{PFS}=$ progression-free survival. 
Table 2 Univariate Analysis: Association Between SNP and Outcome

\begin{tabular}{|c|c|c|c|c|c|}
\hline Variables & No. Patients & Median PFS, mos & $P$ Value & Median OS, mos & $P$ Value \\
\hline \multicolumn{6}{|c|}{ FGFR2 rs2981582 906C>T } \\
\hline No & 134 & 15 & .0007 & 33 & .04 \\
\hline Yes & 20 & 8 & & 22 & \\
\hline \multicolumn{6}{|c|}{ Bone metastases } \\
\hline No & 99 & 14 & .098 & 34 & .01 \\
\hline Yes & 55 & 11 & & 19 & \\
\hline \multicolumn{6}{|c|}{ Neutrophil count $>7800 / \mathrm{mm}^{3}$} \\
\hline No & 139 & 14 & .0001 & 31 & $<.0001$ \\
\hline Yes & 13 & 3 & & 6 & \\
\hline \multicolumn{6}{|c|}{ Platelet count $>450,000 / \mathrm{mm}^{3}$} \\
\hline No & 133 & 14 & .01 & 34 & .0002 \\
\hline Yes & 20 & 7 & & 13.5 & \\
\hline \multicolumn{6}{|c|}{ Sarcomatoid dedifferentiation $\geq 25 \%$} \\
\hline No & 133 & 14 & .0006 & 30 & .008 \\
\hline Yes & 5 & 2 & & 14 & \\
\hline \multicolumn{6}{|c|}{ Karnofsky performance status $\leq 70$} \\
\hline No & 128 & 14 & .007 & 31 & .006 \\
\hline Yes & 25 & 8 & & 14 & \\
\hline \multicolumn{6}{|c|}{$\begin{array}{l}\text { Hemoglobin low }(<12 \mathrm{~g} / \mathrm{dL} \text { [women] } \\
\text { or }<14 \mathrm{~g} / \mathrm{dL} \text { [men]) }\end{array}$} \\
\hline No & 70 & 16 & .097 & 35 & .18 \\
\hline Yes & 83 & 11 & & 23 & \\
\hline \multicolumn{6}{|c|}{$\mathrm{LDH}>1.5 \mathrm{ULN}$} \\
\hline No & 140 & 14 & .11 & 31 & .15 \\
\hline Yes & 9 & 10 & & 22 & \\
\hline \multicolumn{6}{|c|}{ Corrected calcium $>10 \mathrm{mg} / \mathrm{dL}$} \\
\hline No & 137 & 13 & .46 & 30 & .48 \\
\hline Yes & 11 & 21 & & 29 & \\
\hline \multicolumn{6}{|c|}{$\begin{array}{l}\text { Time from nephrectomy to systemic } \\
\text { treatment }<12 \text { mos }\end{array}$} \\
\hline No & 53 & 15 & .08 & 42 & .01 \\
\hline Yes & 100 & 12 & & 27 & \\
\hline
\end{tabular}

In univariate analysis, median PFS and median OS were estimated by Kaplan-Meier, and $P$-values are derived from a log-rank test.

Bold value indicates significance.

Abbreviations: FGFR2 = Fibroblast growth factor receptor 2; LDH = lactate dehydrogenase activity; OS = overall survival; PFS = progression free survival; SNP = single nucleotide polymorphism; ULN = upper limit of normal.

patient characteristics usually associated with prognosis, including IMDC score, were well-balanced between TT and CT/CC carriers, except baseline $\mathrm{LDH}$ (above $1.5 \times$ upper limit of normal: $21 \%$ in TT vs. $4 \%$ in CT/CC carriers; $P=.02$ ). However, median baseline LDH levels were identical in TT and CT/CC carriers (232.0 vs. 247.5 U/L; $P=.8$ ). In the multivariate analysis, rs 2981582 remained as independently associated with PFS with an HR of 2.858 (95\% CI, 1.659-4.923; $P<.0001)$ and with OS with an HR of 1.795 (95\% CI, 1.003-3.212; $P=.049$ ) (Table 3). LDH levels were not associated with PFS (HR 0.677; 95\% CI, 0.240-1.910; $P=.46)$ nor OS (HR, 0.531; 95\% CI, 0.181-1.562; $P=.25$ ). Supplemental Figure 1 (in the online version) shows that the negative impact of the TT variant on PFS can be observed in all IMDC risk group patients. Thus, the poor outcome for TT patients seems not to be driven by the higher frequency of elevated LDH nor by IMDC risk stratification.

\section{Supplementary Internal Validation}

As an additional internal validation of our results, we analyzed the impact of rs2981582 in the subgroup of patients treated at Belgian $(\mathrm{n}=102)$ and at French sites $(\mathrm{n}=52)$ included in this study. An identical significant impact on mPFS was observed in both subgroups (see Supplemental Figure 2 in the online version).

\section{Discussion}

The aim of this study was to investigate the impact of SNP rs2981582 in FGFR2 in patients with mccRCC treated with sunitinib as first-line VEGFR-targeted therapy. 


\section{FGFR2 Polymorphism as Biomarker for Outcome on Sunitinib}

Table 3 Multivariate Analysis for PFS and OS

\begin{tabular}{|c|c|c|c|}
\hline Variable & $P$ Value & Hazard Ratio & 95\% Confidence Interval \\
\hline \multicolumn{4}{|l|}{ Multivariate analysis for PFS } \\
\hline Neutrophil count $>7800 / \mathrm{mm}^{3}$ & .002 & 2.878 & $1.450-5.709$ \\
\hline Platelet count $>450,000 / \mathrm{mm}^{3}$ & .010 & 2.129 & $1.196-3.790$ \\
\hline Karnofsky performance status $\leq 70$ & .013 & 1.965 & $1.150-3.357$ \\
\hline Sarcomatoid dedifferentiation $\geq 25 \%$ & .019 & 3.414 & $1.223-9.532$ \\
\hline FGFR2 rs2981582 TT polymorphism & .000 & 2.858 & $1.659-4.923$ \\
\hline \multicolumn{4}{|l|}{ Multivariate analysis for OS } \\
\hline Neutrophil count $>7800 / \mathrm{mm}^{3}$ & $<.0001$ & 5.774 & $2.806-11.878$ \\
\hline Platelet count $>450,000 / \mathrm{mm}^{3}$ & .001 & 2.619 & $1.447-4.741$ \\
\hline Karnofsky performance status $\leq 70$ & .022 & 1.993 & $1.104-3.598$ \\
\hline Time from nephrectomy to systemic treatment $<12$ mos & .014 & 1.827 & $1.131-2.951$ \\
\hline Bone metastases & .008 & 1.844 & $1.174-2.896$ \\
\hline FGFR2 rs2981582 TT polymorphism & .049 & 1.795 & $1.003-3.212$ \\
\hline
\end{tabular}

Abbreviations: FGFR2 = fibroblast growth factor receptor 2; OS = overall survival; PFS = progression free survival.

In this series of 154 patients, we found a statistically and clinically significant impact of the TT variant on outcome. Compared with patients with the CT/CC genotype, patients with the TT genotype had significantly poorer mPFS and mOS and less important tumor shrinkage on sunitinib. rs2981582 remained as an independent predictor of $\mathrm{mPFS}$ and $\mathrm{mOS}$ on multivariate analysis. The impact of rs2981582 was stronger on PFS than on OS, but we have to consider the impact of subsequent therapy lines, among them immune checkpoint inhibitors, on OS.

Considering merely the correlation with mPFS and mOS, it is impossible to differentiate if the impact of rs 2981582 is prognostic or predictive. The impact of rs 2981582 would be prognostic, if a longer mPFS and $\mathrm{mOS}$ are the consequence of a more indolent disease in CC/CT carriers and a more aggressive disease in TT carriers. The impact of rs 2981582 would be predictive, if a longer mPFS and mOS are the result of an improved efficacy of sunitinib in CC/CT carriers compared with TT carriers. In the latter case, the polymorphism should also be strongly correlated to tumor shrinkage. Based on our findings, although rs2981582 was correlated to median tumor shrinkage, we still cannot state that rs2981582 is a predictive biomarker for response on sunitinib. Indeed, even in TT carriers, partial responses have been noticed in our patient series.

Similar data in literature are scarce. rs2981582 in FGFR2 was previously found to be associated with treatment outcome in patients with mccRCC treated in the first-line with pazopanib. rs 2981582 was associated with mPFS $(P=.053)^{24}$ and with mOS (HR, 1.40; $95 \%$ CI, $1.09-1.81 ; P=.008),{ }^{25}$ favoring CT/CC carriers.

The validation of findings on the prognostic or predictive value of specific SNPs in patients with mccRCC treated with VEGFR-TKIs has been challenging. ${ }^{31}$ The most concordant results were found in SNPs in the efflux pump ABCB1 $1^{17,32-35}$ and in interleukin-8..$^{25,36}$ Findings on the impact of SNPs in VEGFR1 (rs9582036) ${ }^{16}$ and VEGFR3 (rs307826), ${ }^{14,17}$ although similarly shown in independent series, were not confirmed in other patient cohorts. ${ }^{18,19}$ However, findings concerning the impact of rs 2981582 are now coherent in 154 patients treated with sunitinib and 380 with pazopanib, totaling 534 patients. This is an argument in favor of the robustness of these findings, which now should be validated in further independent patient series.

FGFR2 amplifications and mutations have been described in multiple cancer types. ${ }^{37}$ However, FGFR2 mutations are rare in ccRCCs, ${ }^{2}$ and data on FGFR2 amplification are scarce. FGFR2 is located on chromosome 10q26 and encodes a receptor tyrosine kinase that is involved in multiple processes like cell growth, invasiveness, mortality, and VEGF-independent angiogenesis. ${ }^{38}$ FGFR2 amplifications have been reported in up to $10 \%$ of gastric cancers, most of which are diffuse-type with relatively poor prognosis. ${ }^{39}$ In a series of 125 patients with invasive ductal breast carcinoma, a significant association between cytoplasmic FGFR2 expression levels and tumor size was shown. Higher expression levels of FGFR2 were associated with lower OS and disease-free survival. ${ }^{40}$ Finally, the association with rs2981582 and breast cancer susceptibility is another argument in favor of a (patho)physiologic impact of this polymorphism. In a meta-analysis, FGFR2 was confirmed as a breast cancer susceptibility gene, and various variants of FGFR2 are significantly associated with breast cancer risk. For rs2981582, 39 studies for a total of 93,000 patients and 107,000 controls were evaluated. The corresponding odds ratio for developing breast cancer in heterozygous individuals was 1.21 , whereas homozygous individuals (TT) carried an odds ratio of 1.48 compared with people carrying the wild type $(\mathrm{CC})(P<.001) .{ }^{41}$

The TT polymorphism in rs2981582 906C $>\mathrm{T}$ leads to increased transcription and expression of FGFR2 ${ }^{23}$ and thus possibly to increased VEGF-independent angiogenesis. When VEGF inhibitors successfully block angiogenetic pathways that rely on VEGF, other pro-angiogenic factors and pathways, such as the FGF-FGFR-axis, can be activated and be responsible for further vessel growth and disease progression (kinase switch theory). The result is a stimulation of endothelial cell, fibroblast, and tumor cell growth and function. Unfortunately, FGFR2 mRNA-expression data were not available. However, most probably, it will not be FGFR2-expression in the primary kidney tumor, but in metastases resisting to sunitinib that could be correlated to the 
FGFR2-genotype. Unfortunately, tissue samples of metastases resisting to systemic therapy are usually only rarely available.

FGFR inhibitors such as lenvatinib and dovitinib have been tested in mccRCC in clinical studies. Lenvatinib is a TKI targeting FGFR1, 2, 3, and 4 and VEGFR. Lenvatinib was tested in a phase II trial in patients progressing on a previous VEGF-targeted therapy. Patients received lenvatinib and the mammalian target of rapamycin inhibitor everolimus or single-agent treatment with these 2 agents. Lenvatinib plus everolimus or lenvatinib alone resulted in a PFS benefit compared with everolimus in monotherapy. The RR was $43 \%$ in patients receiving lenvatinib plus everolimus, compared with $6 \%$ in patients receiving everolimus in monotherapy. This RR with lenvatinib was higher than the RR usually seen in second-line VEGFR-TKIs. ${ }^{22,42}$ Dovitinib is a TKI targeting, besides the VEGFR, also FGFR1 and FGFR3. Dovitinib was tested in a phase III study as a third-line therapy in patients with mccRCC treated in the first-line with VEGF-targeted therapy and in the second-line with everolimus. Patients were randomized between dovitinib and sorafenib. Surprisingly, mPFS and mOS were similar in both treatment arms, and the study was considered negative. The results of this study have challenged the hypothesis that resistance to antiVEGF-TKIs is mainly owing to FGFR activation. ${ }^{21}$ Possibly, the difference in efficacy between lenvatinib and dovitinib can be explained by a larger FGFR-inhibition by lenvatinib.

Our pharmacogenomics study has several potential limitations. First, it was a retrospective, uncontrolled analysis of patients treated in several centers without a central protocol dictating the treatment schedule and dose modifications or the timing of radiologic assessments. Second, because our patients were mainly Caucasian, the relevance of these polymorphisms needs to be assessed in other ethnic groups because of possible genetic heterogeneity. Finally, at this moment, these findings cannot be used for patient selection for treatment with VEGFR-TKIs. However, these results provide further evidence that FGFR2 is involved in resistance to VEGFRTKIs in patients with mccRCC.

\section{Conclusions}

Polymorphism rs2981582 in FGFR2 is correlated to outcome in patients with mccRCC treated with sunitinib. The TT genotype is associated with poorer PFS, poorer OS, and reduced target lesion shrinkage during treatment compared with the CC and CT genotypes. Prospective validation of this SNP is now warranted.

\section{Clinical Practice Points}

- Biomarkers predicting outcome on VEGFR-TKIs in mccRCC are lacking.

- We have found rs2981582, a polymorphism in the FGFR2, to be a potential biomarker associated with PFS and OS on the VEGFR-TKI sunitinib in mccRCC.

- In our series of 154 patients, TT variant carriers had a poorer outcome compared with CT/CC carriers: the mPFS was 8 versus 15 months $(P=.0007)$, and the mOS was 22 versus 33 months $(P=.04)$, respectively. Moreover, the median shrinkage of selected tumor target lesions during treatment with sunitinib was $-16 \%$ versus $-31 \%(P=.002)$. On multivariate analysis, rs2981582 remained an independent predictor of PFS and OS.
Previously, the same impact was shown in patients with mccRCC treated with the VEGFR-TKI pazopanib. TT variant carriers might have increased angiogenesis through the FGFR2pathway, leading to escape of the tumor when treated with sunitinib or pazopanib.

- These findings, when validated, might have a clinical impact in the future: they could be used for patient counseling on prognosis and might also explain the efficacy of FGFR blockers in mccRCC.

\section{Acknowledgments}

B. Beuselinck received a grant from the Fondation Martine Midy (Paris, France) (2009-2010), the Fonds voor Wetenschappelijk Onderzoek Vlaanderen (Belgium) (2011-2013), and an unrestricted research grant from Pfizer (2014). D. Lambrechts is supported by the Stichting Tegen Kanker. E. Lerut received funds from Fonds voor Wetenschappelijk Onderzoek Vlaanderen (Belgium) and Stichting tegen Kanker. A. Verbiest received an Emmanuel van der Schueren grant from Stichting Kom op tegen Kanker.

\section{Disclosure}

S. Oudard received honoraria from Novartis, Pfizer, Roche, BMS, and Bayer. P. Schöffski received an institutional travel grant from Pfizer related to clinical research in non-clear-cell renal cell carcinoma. J.J. Patard is a consultant and principal investigator in Pfizer trials. D. Lambrechts served on advisory boards from Roche, Sanofi, Bayer, Novartis, Boehringer, and Eli-Lilly and received honoraria for this. B. Beuselinck received honoraria from Amgen, Pfizer, Janssen, Ipsen, and Bayer. B. Beuselinck is an investigator of the EudraCT: 2011-006085-40/METASUN trial supported by Pfizer, and he received honoraria from Ipsen, Amgen, Novartis, and Pfizer. The other authors have no conflicts of interest to declare.

\section{Supplemental Data}

Supplemental figures accompanying this article can be found in the online version at https://doi.org/10.1016/j.clgc.2018.11.002.

\section{References}

1. Sato Y, Yoshizato T, Shiraishi Y, et al. Integrated molecular analysis of clear-cell renal cell carcinoma. Nat Genet 2013; 45:860-7.

2. Cancer Genome Atlas Research Network. Comprehensive molecular characterization of clear cell renal cell carcinoma. Nature 2013; 499:43-9.

3. Fakih M, Schiff D, Erlich R, Logan TF. Intramedullary spinal cord metastasis (ISCM) in renal cell carcinoma: a series of six cases. Ann Oncol 2001; 12:1173-7.

4. Motzer RJ, Hutson TE, Tomczak P, et al. Overall survival and updated results for sunitinib compared with interferon alfa in patients with metastatic renal cell carcinoma. J Clin Oncol 2009; 27:3584-90.

5. Motzer RJ, Hutson TE, Cella D, et al. Pazopanib versus sunitinib in metastatic renal-cell carcinoma. $N$ Engl J Med 2013; 369:722-31.

6. Motzer RJ, Hutson TE, Tomczak P, et al. Sunitinib versus interferon alfa in metastatic renal-cell carcinoma. $N$ Engl J Med 2007; 356:115-24.

7. Sternberg CN, Davis ID, Mardiak J, et al. Pazopanib in locally advanced or metastatic renal cell carcinoma: results of a randomized phase III trial. J Clin Oncol 2010; 28:1061-8.

8. McKay RR, Kroeger N, Xie W, et al. Impact of bone and liver metastases on patients with renal cell carcinoma treated with targeted therapy. Eur Urol 2014; 65: 577-84.

9. Kim BJ, Kim JH, Kim HS, Zang DY. Prognostic and predictive value of VHL gene alteration in renal cell carcinoma: a meta-analysis and review. Oncotarget 2017; 8:13979-85.

10. You D, Song SH, Cho YM, et al. Predictive role of tissue-based molecular markers in patients treated with sunitinib for metastatic renal cell carcinoma. World J Urol 2015; 33:111-8. 


\section{FGFR2 Polymorphism as Biomarker for Outcome on Sunitinib}

11. Garcia-Donas J, Leandro-Garcia LJ, Gonzalez Del Alba A, et al. Prospective study assessing hypoxia-related proteins as markers for the outcome of treatment with sunitinib in advanced clear-cell renal cell carcinoma. Ann Oncol 2013; 24:2409-14.

12. Beuselinck B, Verbiest A, Couchy G. Pro-angiogenic gene expression is associated with better outcome on sunitinib in metastatic clear-cell renal cell carcinoma. Acta Oncol 2018; 57:498-508.

13. Beuselinck B, Job S, Becht E, et al. Molecular subtypes of clear cell renal cell carcinoma are associated with sunitinib response in the metastatic setting. Clin Cancer Res 2015; 21:1329-39.

14. Garcia-Donas J, Esteban E, Leandro-García LJ, et al. Single nucleotide polymorphism associations with response and toxic effects in patients with advanced renal-cell carcinoma treated with first-line sunitinib: a multicentre, observational, prospective study. Lancet Oncol 2011; 12:1143-50.

15. Dornbusch J, Walter M, Gottschalk A, et al. Evaluation of polymorphisms in angiogenesis-related genes as predictive and prognostic markers for sunitinib-treated metastatic renal cell carcinoma patients. J Cancer Res Clin Oncol2016; 142:1171-82.

16. Beuselinck B, Jean-Baptiste J, Schoffski P, et al. Validation of VEGFR1 rs 9582036 as predictive biomarker in metastatic clear-cell renal cell carcinoma patients treated with sunitinib. BJU Int 2016; 118:890-901.

17. Beuselinck B, Karadimou A, Lambrechts D, et al. Single-nucleotide polymorphisms associated with outcome in metastatic renal cell carcinoma treated with sunitinib. Br J Cancer 2013; 108:887-900.

18. Liu X, Swen JJ, Boven E, et al. Meta-analysis on the association of VEGFR1 genetic variants with sunitinib outcome in metastatic renal cell carcinoma patients. Oncotarget 2017; 8:1204-12.

19. Motzer RJ, Hutson TE, Hudes GR, et al. Investigation of novel circulating proteins, germ line single-nucleotide polymorphisms, and molecular tumor markers as potential efficacy biomarkers of first-line sunitinib therapy for advanced renal cell carcinoma. Cancer Chemother Pharmacol 2014; 74:739-50.

20. Casanovas O, Hicklin DJ, Bergers G, Hanahan D. Drug resistance by evasion of antiangiogenic targeting of VEGF signaling in late-stage pancreatic islet tumors. Cancer Cell 2005; 8:299-309.

21. Motzer RJ, Porta C, Vogelzang NJ, et al. Dovitinib versus sorafenib for third-line targeted treatment of patients with metastatic renal cell carcinoma: an open-label, randomised phase 3 trial. Lancet Oncol 2014; 15:286-96.

22. Motzer RJ, Hutson TE, Glen H, et al. Lenvatinib, everolimus, and the combination in patients with metastatic renal cell carcinoma: a randomised, phase 2, open-label, multicentre trial. Lancet Oncol 2015; 16:1473-82.

23. Meyer KB, Maia AT, O'Reilly M, et al. Allele-specific up-regulation of FGFR2 increases susceptibility to breast cancer. PLoS Biol 2008; 6:e108.

24. Xu CF, Bing NX, Ball HA, et al. Pazopanib efficacy in renal cell carcinoma: evidence for predictive genetic markers in angiogenesis-related and exposure-related genes. J Clin Oncol 2011; 29:2557-64.

25. Xu CF, Johnson T, Garcia-Donas J, et al. IL8 polymorphisms and overall survival in pazopanib- or sunitinib-treated patients with renal cell carcinoma. Br J Cancer 2015; 112(Suppl):1190-8.

26. Reumers J, De Rijk P, Zhao H, et al. Optimized filtering reduces the error rate in detecting genomic variants by short-read sequencing. Nat Biotechnol 2012; 30:61-8.
27. Heng DY, Xie W, Regan MM, et al. Prognostic factors for overall survival in patients with metastatic renal cell carcinoma treated with vascular endothelial growth factor-targeted agents: results from a large, multicenter study. J Clin Oncol 2009; 27:5794-9.

28. Verbiest A, Couchy G, Job S, et al. Molecular subtypes of clear-cell renal cell carcinoma are prognostic for outcome after complete metastasectomy. Eur Urol 2018; 74:474-80.

29. Verbiest A, Couchy G, Job S, et al. Molecular subtypes of clear cell renal cell carcinoma are associated with outcome during pazopanib therapy in the metastatic setting. Clin Genitourin Cancer 2018; 16:e605-12.

30. Gore ME, Szczylik C, Porta C, et al. Final results from the large sunitinib global expanded-access trial in metastatic renal cell carcinoma. $\mathrm{Br} J$ Cancer 2015; 113:12-9.

31. Beuselinck B, Zucman-Rossi J. Kidney cancer: Single nucleotide polymorphisms in mRCC-is their time up? Nat Rev Urol 2015; 12:424-6.

32. Diekstra MH, Swen JJ, Boven E, et al. CYP3A5 and ABCB1 polymorphisms as predictors for sunitinib outcome in metastatic renal cell carcinoma. Eur Urol 2015; 68:621-9.

33. van der Veldt AA, Eechoute K, Gelderblom H, et al. Genetic polymorphisms associated with a prolonged progression-free survival in patients with metastatic renal cell cancer treated with sunitinib. Clin Cancer Res 2011; 17:620-9.

34. Chu YH, Li H, Tan HS, et al. Association of ABCB1 and FLT3 polymorphisms with toxicities and survival in Asian patients receiving sunitinib for renal cell carcinoma. PLoS One 2015; 10:e0134102.

35. Beuselinck B, Lambrechts D, Van Brussel T, et al. Efflux pump ABCB1 single nucleotide polymorphisms and dose reductions in patients with metastatic renal cell carcinoma treated with sunitinib. Acta Oncol 2014; 53:1413-22.

36. Motzer RJ, Figlin RA, Martini JF, et al. Germline genetic biomarkers of sunitinib efficacy in advanced renal cell carcinoma: results from the RENAL EFFECT trial. Clin Genitourin Cancer 2017; 15:526-33.

37. Brooks AN, Kilgour E, Smith PD. Molecular pathways: fibroblast growth factor signaling: a new therapeutic opportunity in cancer. Clin Cancer Res 2012; 18: 1855-62.

38. Turner N, Grose R. Fibroblast growth factor signalling: from development to cancer. Nat Rev Cancer 2010; 10:116-29.

39. Kunii K, Davis L, Gorenstein J, et al. FGFR2-amplified gastric cancer cell lines require FGFR2 and Erbb3 signaling for growth and survival. Cancer Res 2008; 68: 2340-8.

40. Sun S, Jiang Y, Zhang G, et al. Increased expression of fibroblastic growth factor receptor 2 is correlated with poor prognosis in patients with breast cancer. J Surg Oncol 2012; 105:773-9.

41. Cui F, Wu D, Wang W, He X, Wang M. Variants of FGFR2 and their associations with breast cancer risk: a HUGE systematic review and meta-analysis. Breast Cancer Res Treat 2016; 155:313-35.

42. Sacre A, Barthelemy P, Korenbaum C, et al. Prognostic factors in second-line targeted therapy for metastatic clear-cell renal cell carcinoma after progression on an anti-vascular endothelial growth factor receptor tyrosine kinase inhibitor. Acta Oncol 2016; 55:329-40. 


\section{Supplemental Data}

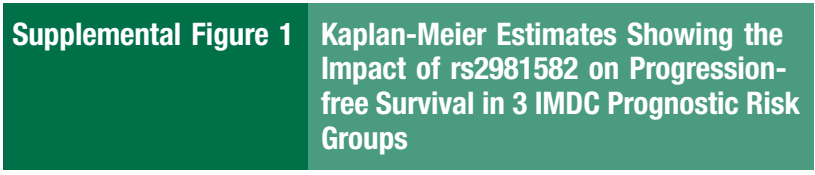

PFS (\%)

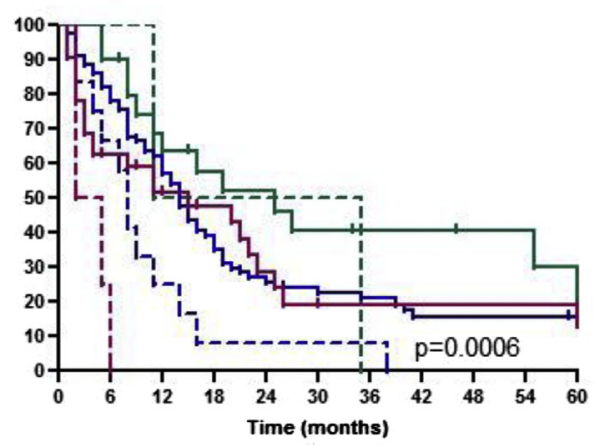

- IMDC good risk CC+CT (25 months)

-r. IMDC good risk TT (23 months)

- IMDC intermediate risk CC+CT (14 months)

-r. IMDC intermediate risk TT (8 months)

+ IMDC poor risk CC+CT (15 months)

-r. IMDC poor risk TT (3.5 months)

Abbreviations: IMDC = International Metastatic Renal Cell Carcinoma Database Consortium; $\mathrm{PFS}=$ progression-free survival 


\section{FGFR2 Polymorphism as Biomarker for Outcome on Sunitinib}

Supplemental Figure 2 Internal Validation: Kaplan-Meier Estimates Showing the Same Impact of rs2981582 on Progression-free Survival in Belgian (A) and French (B) Patients

A

PFS (\%) BELGIAN PATIENTS

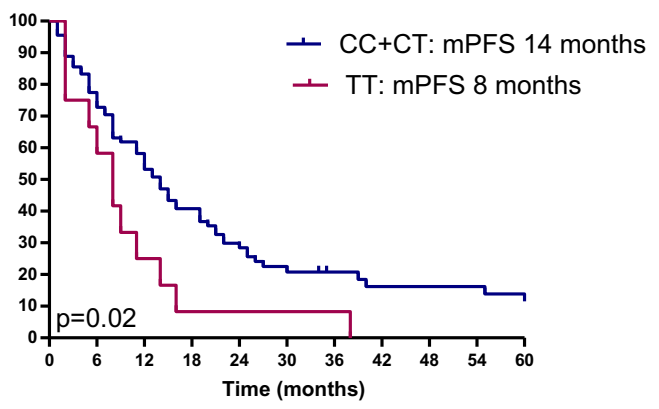

Months
CC/CT

$\begin{array}{lllllll}0 & 6 & 12 & 18 & 24 & 30 & 36 \\ 90 & 66 & 47 & 30 & 20 & 13 & 9 \\ 12 & 8 & 3 & 1 & 1 & 1 & 1\end{array}$
B PFS (\%) FRENCH PATIENTS

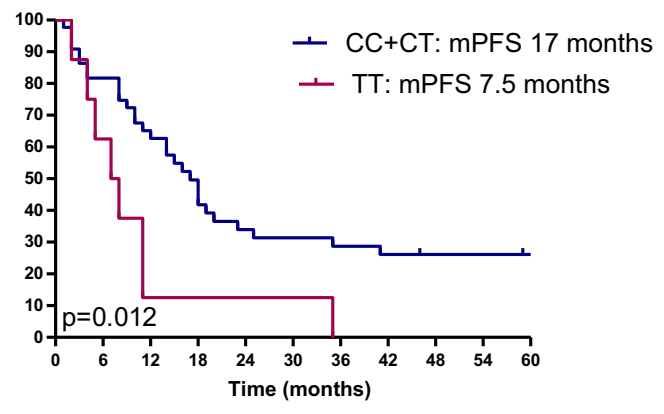

Months
CC/CT

Abbreviations: $m=$ median; PFS = progression-free survival. 